\title{
Towards quantitative molecular mapping of cells by Raman microscopy: using AFM for decoupling molecular concentration and cell topography
}

\author{
Radu Boitor ${ }^{1}$, Faris Sinjab ${ }^{1}$, Stephanie Strohbuecker ${ }^{2}$, Virginie Sottile ${ }^{2}$, Ioan Notingher ${ }^{1}$ * \\ ${ }^{1}$ School of Physics and Astronomy, University of Nottingham, University Park, Nottingham NG7 2RD, U.K. \\ ${ }^{2}$ Wolfson STEM Centre, School of Medicine, University of Nottingham, University Park, Nottingham NG7 2RD, \\ U.K. \\ *corresponding author: ioan.notingher@nottingham.ac.uk
}

\begin{abstract}
Raman micro-spectroscopy (RMS) is a non-invasive technique for imaging live cells in-vitro. However, obtaining quantitative molecular information from the Raman spectra is difficult because the intensity of a Raman band is proportional to the number of molecules in the sampled volume, which depends on the local molecular concentration and the thickness of the cell. In order to understand these effects, we combined RMS with atomic force microscopy (AFM), a technique that can measure accurately the thickness profile of the cells. Solution-based calibration models for RNA and albumin were developed to create quantitative maps of RNA and proteins in individual fixed cells. The maps were built by applying the solution-based calibration models, based on partial least square fitting (PLS), on raster-scan Raman maps, after accounting for the local cell height obtained from the AFM. We found that concentrations of RNA in the cytoplasm of mouse neuroprogenitor stem cells (NSCs) were as high as $25 \pm 6 \mathrm{mg} / \mathrm{m}$, while proteins were distributed more uniformly and reaching concentrations as high as $\sim 50 \pm 12$ $\mathrm{mg} / \mathrm{ml}$. The combined AFM-Raman datasets from fixed cells were also used to investigate potential improvements for normalization of Raman spectral maps. For all Raman map of fixed cells $(n=10)$, we found a linear relationship between the scores corresponding to the first component (PC1) and cell height profile obtained by AFM. We used PC1 scores to reconstruct the relative height profiles of independent cells $(n=10)$, and obtained correlation coefficients with AFM maps higher than 0.99. Using this normalization method, qualitative maps of RNA and protein were obtained concentrations for live NSCs. While this study demonstrates the potential of using AFM and RMS for measuring concentration maps for individual NSCs in-vitro, further studies are required to establish the robustness of the normalization method based on principal component analysis when comparing Raman spectra of cells with large morphological differences.
\end{abstract}




\section{Introduction}

Cells are the fundamental units of any living organism. Their functions rely on highly orchestrated, spatially- and time-dependent molecular interactions that underpin all biological processes. However, because of their small size and fragile nature, non-invasive detection and quantification of molecular interactions in living cells has always been a challenge. Various techniques for quantitative molecular analysis of cells do exist (e.g. mass spectrometry [1], polymerase chain reaction (PCR) [2]), yet they require destructive procedures and usually apply to whole cell populations instead of single cells. Therefore, these techniques provide only single time-points and cannot provide insight into discrete and dynamic molecular events in living cells. Similar limitations apply to microscopy techniques based on fluorescence labeling of cells. As most molecules of interest are found inside the cells, cell fixation and membrane permeabilisation are required in order to allow the fluorescent molecules, which often are large dye molecules, to enter the cell cytoplasm and attach to the molecules of interest. To circumvent this antibody-based detection approach, transgenic strategies to express markers such as green fluorescent protein (GFP) have been developed. However, these techniques cannot be readily applied to primary cells, and genetic manipulation of cells requires laborious protocols to be developed for each cell type. Another limitation common to all fluorescence imaging methods is the difficulty in quantifying the results due to intensity variations of the fluorescence emission caused by photo-bleaching as well as variations in staining protocols.

Raman micro-spectroscopy (RMS) is a well-established analytical technique based on interaction of light with molecules present in the sample. Research during the last two decades showed that RMS can provide detailed molecular information of complex biological samples such as tissues and cells [3]. The main advances in this field have been recently reviewed [4-6]. An important feature of RMS is that it can measure time- and spatially-resolved molecular processes in live cells maintained under physiological condition in-vitro, and without requiring labeling [7-10].

Recently, attempts have been reported for quantitative molecular analysis of individual cells, as well as mapping the concentration of molecular components in live cells. The use of Raman spectral calibration models developed using samples with known concentrations (micro-particles or solutions) has been reported for quantifying glycogen content in stem cell populations [11] and RNA in neuroprogenitor stem cells [12]. However, one main challenge when attempting such quantitative analysis arises from the fact that the intensity of a Raman band is proportional to the number of molecules in the sampled volume, which depends on the local concentration and the local thickness of the cell, as well as the laser focusing conditions. Furthermore, most biological processes are also accompanied by morphological changes of the cells. Therefore, it is often difficult to extract and quantify the molecular concentration in live cells because the 3D morphology of the cells is not known (Figure 1). 


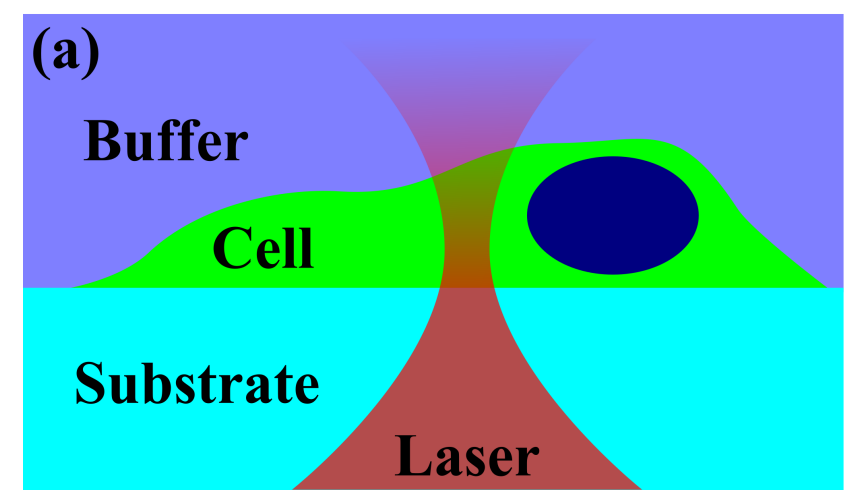

(b)

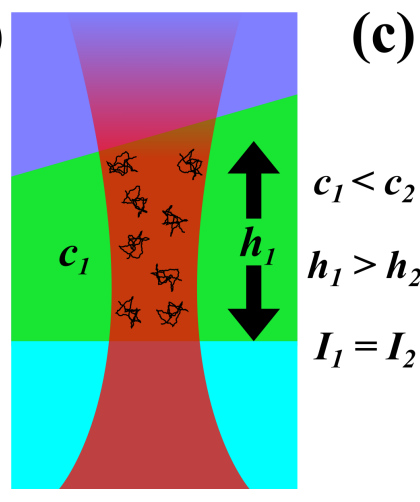

(c)

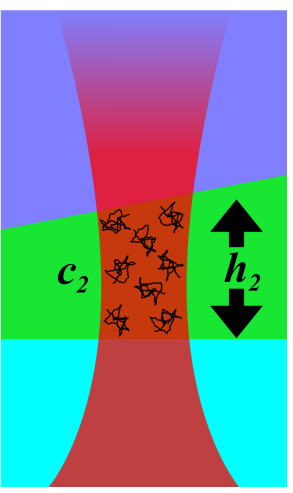

Figure 1. Coupling between concentration and cell thickness in Raman micro-spectroscopy measurements. Schematic of the Raman sampling geometry for cells is shown in (a). The situations shown in (b) and (c) will give the same Raman scattered intensity, despite different molecular concentrations.

When attempting to discriminate between two groups of cells based on their Raman spectra, it is common to normalize the Raman spectra, either using the intensity of certain bands that are expected to remain constant (e.g. $1450 \mathrm{~cm}^{-1}$ [13] or $1004 \mathrm{~cm}^{-1}$ [14]), the total area under the baseline-corrected spectrum [15,16], or using the standard normal variance method [17-19]. Such normalization procedures have also been combined with solution-based calibration models to obtain molecular maps (e.g. RNA) in individual cells [12]. However, no studies on the validity of these normalization methods have been reported to date, thus the ability of these techniques to truly account for the morphology of the cells remains unclear.

In this paper we used confocal Raman micro-spectroscopy in conjunction with atomic force microscopy (AFM) in order to separate the effects of cell morphology and molecular concentration in the measured Raman spectra of cells. AFM was used to measure the topography maps of each individual cell, and this information was used to correct the intensity of the Raman spectra. Although in principle the topography of the cell could be obtained by recording a 3D map using a confocal Raman microscope, such measurements are time-consuming, and are prone to errors due to uncertainties related to the sampling volume of the microscope (variations in refractive index within the cell and the interfaces at the cell surface). Here, we use solution-based calibration models for proteins and nucleic acids, and combined AFM - Raman mapping to obtain quantitative molecular concentration maps for fixed cells (neuroprogenitor stem cells, NSCs). We also observed that principal component analysis (PCA) may be used for normalization of Raman spectral maps to take into account variations in cell thickness. This normalization method may be particularly useful for normalizing the Raman spectra of live cells, since AFM measurements are typically impractical because of long acquisition times for images and cell motility.

\section{Materials and methods}

\section{Cell Culture}


Cell culture reagents were from Life Technologies (UK) unless otherwise stated. Mouse neural stem cells were cultured as previously described [12]. Briefly, cells were maintained in NSC medium made of DMEM/F12 and Neurobasal medium (1:1), N2, B27, antibiotics and supplemented with bFGF and EGF (Sigma-Aldrich, UK) at 20ng/ml each. Cells were seeded onto low Matrigel-coated chambers (Becton Dickinson, UK) at $10^{5}$ cells $/ \mathrm{ml}$ using Accutase followed by a PBS wash. Cells were fixed with 4\% paraformaldehyde (PFA, VWR) where indicated.

\section{Atomic force microscopy}

The cells that were fixed were washed in phosphate buffer saline (PBS) in order to remove cell debris caused by fixation with PFA. AFM images were acquired with a Nanowizard II AFM (JPK, Germany) that was mounted on top of an inverted Olympus IX-71 microscope. The measurements were performed in tapping mode to minimize the possibility of damage to the cell membrane. Custom made sample holders were built with a thin $(0.17 \mathrm{~mm}) \mathrm{MgF}_{2}$ window on the bottom, that would easily allow access to the microscope objective, but that would also allow space for the AFM scan head from above. Liquid-mode AFM tips (Multi75GB-G, BudgetSensors) were used for all measurements.

\section{Raman micro-spectroscopy}

The sample holders containing the cells were moved to a home- built Raman instrument, which was based on another inverted Olympus IX-71 microscope with an Olympus water immersion 60x objective (NA 1.2, a droplet of water was placed between the objective and coverslip) and with an automated stepper-motor stage (Prior Scientific) used to raster scan the sample, using custom-built software. A $785 \mathrm{~nm}$ Ti:Sapphire laser beam was focused to a laser spot which provided $230 \mathrm{~mW}$ of power at the sample. The Raman back-scattered light was directed to a spectrometer (Andor Shamrock 303i with iDus CCD) via a fiber connection to obtain the Raman spectra. The spectrometer was calibrated using a polystyrene sample using peaks in the fingerprint region. Each fixed cell was scanned for 20 to 60 minutes, depending on its size. For Raman mapping, the integration time for each spectrum was 3 seconds and the step size in $\mathrm{X}, \mathrm{Y}$ directions was $1 \mu \mathrm{m}$. For the axial scans, Raman spectra were recorded at regular $1.1 \mu \mathrm{m} \mathrm{z}$ intervals.

Live cell Raman measurements were performed on the same setup but with different experimental parameters. In order to facilitate live cell measurements, the setup was also equipped with an environmental enclosure (Solent Scientific), which kept the cells under physiological conditions during the measurements $\left(37{ }^{\circ} \mathrm{C}, 5 \% \mathrm{CO}_{2}\right)$. The same $785 \mathrm{~nm}$ laser line was used as for fixed cells, but the power was decreased to $170 \mathrm{~mW}$ and the acquisition time was reduced to $1 \mathrm{~s}$. These parameters have been shown to have a minimal impact on cell physiology, while at the same time providing usable Raman spectra [12].

\section{Raman calibration models}

Quantification of the two investigated cellular components was performed with the use of partial least square regression (PLS). In order to build a PLS calibration model from Raman spectra, 14 
known concentration solutions of RNA (yeast tRNA, lyophilized powder, Sigma UK) and protein (bovine serum albumin, lyophilized powder, Sigma UK) were created. All solutions were created by dissolving the two analytes in high purity deionized water (resistivity of $18.2 \mathrm{M} \Omega \mathrm{cm}$ ). In order to ensure the best correlation between the calibration model and the spectra of fixed cells, the same experimental parameters were used as for the acquisition of spectra from fixed cells: laser power at the sample was $230 \mathrm{~mW}$ and the acquisition time for each spectrum was 3 seconds. The Raman spectra of the calibration samples were measured using of a $6 \mu \mathrm{m}$ thick spacers (Omni-cell spacers from Specac).

\section{Data processing}

All data processing was carried out using custom built MATLAB functions. Cosmic ray spikes were automatically removed from each spectrum before processing. Singular-value decomposition (SVD) was used to remove noise from the spectra whilst preserving $80-90 \%$ of the spectral information. Principal component analysis (PCA) was also used for the quick identification of various cell components and for the removal of the background (composed of contributions from the substrate, objective and the medium surrounding the cell). For the $\mathrm{z}$ profile data, PC1 values at different relative z-positions had a linear baseline subtracted and were offset to 0 minimum value before fitting a Gaussian curve. The top-hat function representing the AFM height, used for de-convolution with the PC1 z-profile had a width specified to an accuracy of $0.1 \mu \mathrm{m}$. The background was linearly subtracted from each spectrum used in the PLS calibration and prediction, in order to limit the spectral features to the ones that are specific to each investigated cellular component. For these spectra, the background was identified automatically with the use of k-means clustering analysis.

For AFM image processing, the background was leveled by fitting a linear plane between 3 points outside the cells and subtracting it from the dataset. In order to correlate the AFM images with the Raman maps the higher resolution AFM images were transformed and cropped in an automated routine to align the AFM image with the frame of the Raman image. The resolution of the resulting AFM image was then reduced to match that of the Raman maps, allowing the data from each to be approximately matched point-for-point.

\section{Results and discussion}

Figure 2 presents typical examples of AFM and Raman spectral images for a fixed NSC. Figure 2(b) shows that the selected Raman spectra at various positions inside the cell represent the molecular composition of the NSC and indicate a high chemical heterogeneity. Raman bands associated to nucleic acids include backbone vibrations at $788 \mathrm{~cm}^{-1}(\mathrm{O}-\mathrm{P}-\mathrm{O})$ and $1095 \mathrm{~cm}^{-1}\left(\mathrm{PO}_{2^{-}}\right.$ ), while vibrations specific to nucleotides are detected at $782 \mathrm{~cm}^{-1}$ (thymine, cytosine and uracil) and $1578 \mathrm{~cm}^{-1}$ (guanine and adenine) [20,21]. In agreement with previous reports, spectra with an intense band assigned to RNA $\left(813 \mathrm{~cm}^{-1}\right.$, typically assigned to O-P-O vibrations in singlestranded nucleic acids) were observed at positions inside the cytoplasm of the NSC cells [12]. Raman bands corresponding to proteins are found in the ranges $1660-1670 \mathrm{~cm}^{-1}$ (Amide I), 1450 $\mathrm{cm}^{-1}$ (C-H bending), 1200-1300 cm (Amide III), $1005 \mathrm{~cm}-1$ (phenylalanine), $854 \mathrm{~cm}^{-1}$ 
(tyrosine) and $760 \mathrm{~cm}^{-1}$ (tryptophan) [22]. All lipids are characterized by intense Raman bands at $1449 \mathrm{~cm}-1$ (C-H bending vibrations), $1301 \mathrm{~cm}^{-1}$ ( $\mathrm{CH}_{2}$ twisting), 1000-1100 $\mathrm{cm}^{-1}$ spectral range (C-C stretching), while Raman bands characteristic to unsaturated lipids can be found at 1658 $\mathrm{cm}^{-1}$ ( $\mathrm{C}=\mathrm{C}$ stretching) [23]. Membrane phospholipids also exhibit Raman bands in the 700-900 $\mathrm{cm}^{-1}$ spectral range assigned to different residues at the phosphate-ester head-group [23]. Highresolution Raman spectral images of cells can be created by plotting the scores obtained after principal component analysis (PCA) of all Raman spectra (Figure 2(d)). These images can identify tentatively cellular structures, such as the nucleus (PC2), endoplasmic reticulum - a lipid/protein rich region surrounding the nucleus (PC2), as well as the RNA rich regions at the edges of the cells (PC3). Indeed, bands corresponding to the molecular species found in these regions can be identified in the PCA loadings (Figure $2(\mathrm{~d})$ ).

Nevertheless, PCA analysis can provide only qualitative information regarding molecular composition of cells. To obtain quantitative information from the Raman spectra, calibration models based on water solutions of RNA and albumin (model for proteins) were developed (Raman spectra of presented in Supplementary Information Figure S1). To reduce the uncertainties related to the laser depth of field, the calibration solutions were placed in a samplechamber of $6 \mu \mathrm{m}$ thickness in between two MgF2 coverslips (Figure 3a). As the thickness of the cells was 1-6 $\mu \mathrm{m}$, the spectra recorded using this sample chamber are more representative for the Raman spectra of cells.
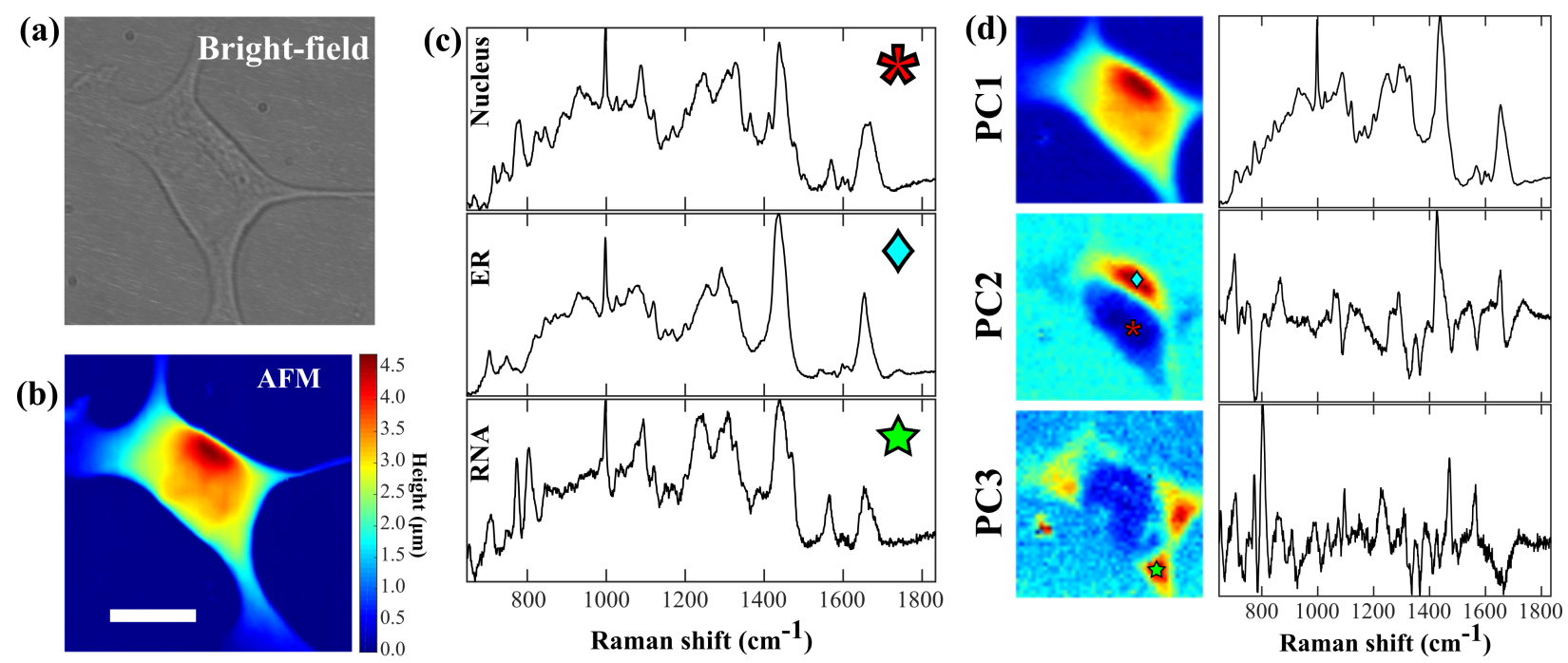

Figure 2. Bright-field (a) and AFM (b) images of the same NSC. Typical spectra from regions indicated by markers in PC score images (c) (SVD from PCs 2:10). (d) PCA images and loadings.

The PLS calibration models were built using fourteen RNA and albumin solutions with known concentrations spaced evenly over the expected concentrations in cells [24]. For the RNA solutions, the calibration model provided a good linear fit with an $\mathrm{R}^{2}$ value of 0.961 . Given the 
relatively low number of samples used for calibration, the prediction performance of the model was estimated by leave-one-out cross validation. When the first four components were used for the PLS model, the root mean squared error for cross-validation was RMSECV $=2.18 \mathrm{mg} / \mathrm{ml}$ (relative error 13\%). Similarly, thirteen Raman spectra of albumin solutions were used for the protein model, producing an $\mathrm{R}^{2}$ value for the calibration curve of 0.942 and a RMSECV of 6.29 $\mathrm{mg} / \mathrm{ml}$ (relative error $15 \%$ ).

(a)

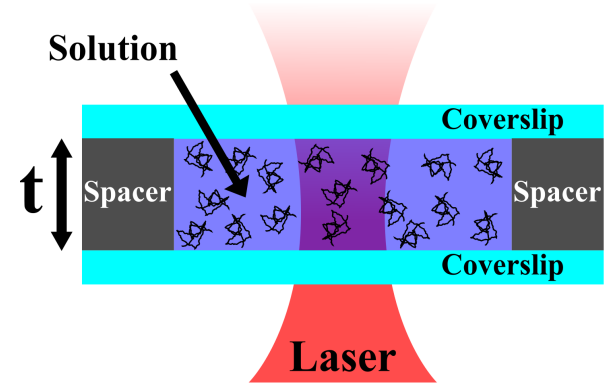

(b)

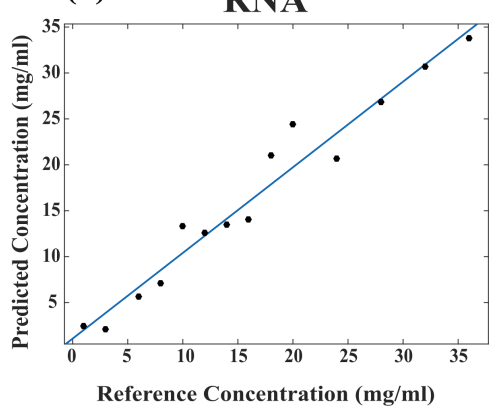

(c)

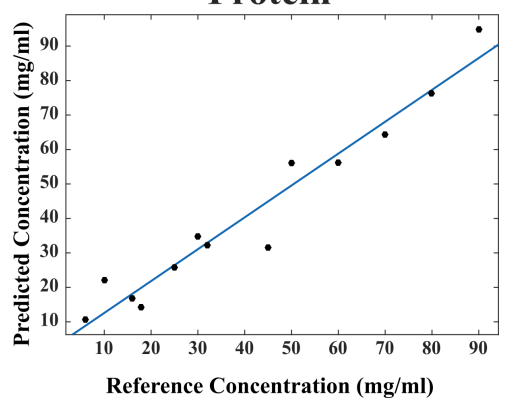

Figure 3. PLS calibration of albumin and RNA. (a) Schematic of the experiment utilizing $t=6$ $\mu \mathrm{m}$ spacers. Calibration curves for RNA (b) and albumin (c) in phosphate buffer saline solutions. The symbols represent the samples used for independent validation.

Next, we applied the solution-based calibrations for RNA and albumin on 2D Raman maps of fixed cells in order to predict the concentration distributions of RNA and proteins. For each pixel in the Raman map $(\sim 1 \mu \mathrm{m} \times 1 \mu \mathrm{m})$, the value of cellular height obtained from the AFM map was used to calculate the local concentrations of RNA and proteins. Figure 4 presents typical examples of quantitative maps for RNA and proteins for fixed NSCs. Individual Raman spectra from selected positions in the cells are presented in the Supplementary Information).

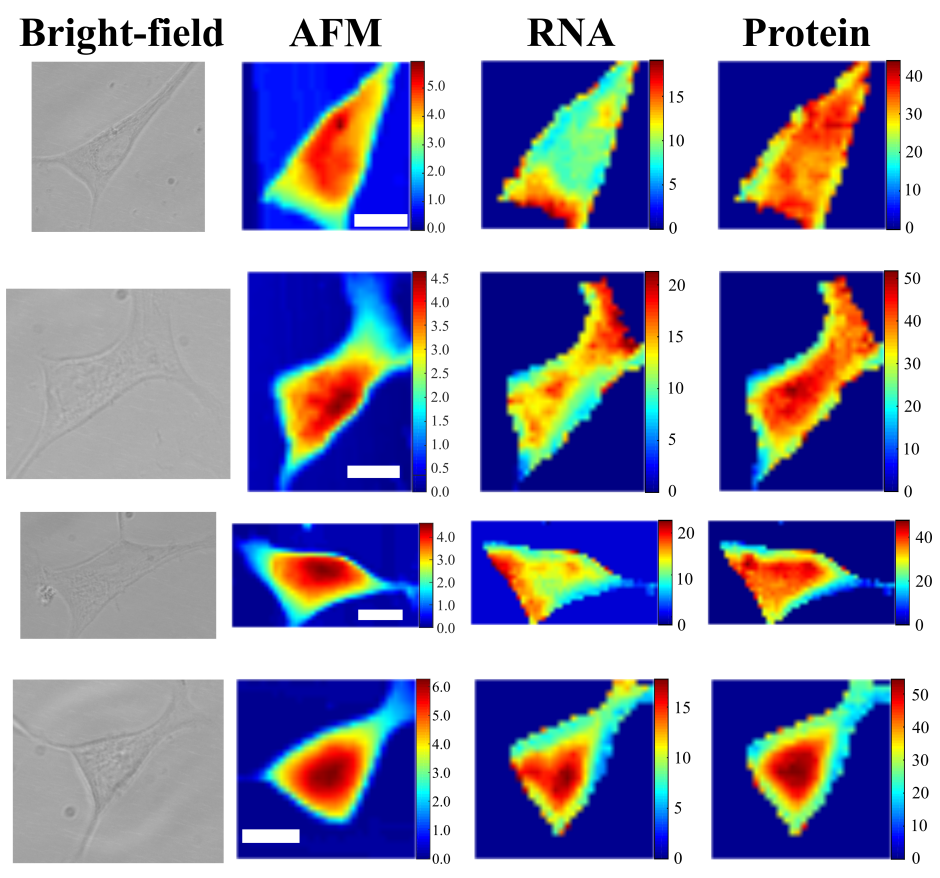


Figure 4. Quantitative Raman mapping. Bright field images, transformed and matched AFM images (scale bar $10 \mu \mathrm{m}$, color scale in $\mu \mathrm{m}$ ) with corresponding concentration maps for RNA and proteins (color scale in $\mathrm{mg} / \mathrm{ml}$ ) for three NSCs.

At each pixel in the concentration maps in Figure 4, the values for the RNA and protein concentrations were calculated by applying the calibration models described in Figure 3 and then scaling using the cell thickness from the AFM map. For cell height values smaller than $1 \mu \mathrm{m}$ in the AFM images, the corresponding Raman spectral intensity approached the limit of detection for the instrument. This resulted in the large values of concentration for the background, thus a threshold was applied based on the AFM height, allowing concentration maps only where cell spectra were detectable by the Raman instrument. When considering the accuracy of the calibration models $(\sim 15 \%)$ and errors in aligning the AFM and Raman maps, we estimate the errors in the concentration maps to be $\sim 25 \%$. These errors may be reduced by increasing the number of samples in the calibration models and by using an integrated AFM-Raman instrument to reduce misalignment between AFM and Raman maps. Co-localized AFM-Raman measurements could eliminate the need for the alignment procedure in post-processing, providing more accurate results.

Figure 4 show that regions with high concentration of RNA can be identified in the cytoplasm of NSCs, with maximum concentrations of typically $25 \pm 6 \mathrm{mg} / \mathrm{ml}$, consistently between cells. We found that the concentration of protein can reach values as high as $50 \pm 12 \mathrm{mg} / \mathrm{ml}$, and were more widespread throughout the cell. The AFM and Raman spectral maps can also be used to quantify the total quantity of RNA and proteins in a single cell. As such, for typical NSC, the total quantity of RNA was found to be $12 \mathrm{pg}$ (with values ranging from $11.1 \mathrm{pg}$ to $13.1 \mathrm{pg}$ ), and $35 \mathrm{pg}$ for proteins respectively (values again ranging in the $32.8-40.2 \mathrm{pg}$ ). The sensitivity level of Raman microscopy compares favorably with other single-cell analytical technique, such as mass spectrometry and PCR. Real-time PCR (RT-PCR) is routinely used for quantification of microRNAs from cell cytoplasm, and achieves detection levels of $\sim 15$ pg of RNA [25]. Mass spectrometry (MS) is a very sensitive analysis technique that allows analysis of even single protein [26]. However, a key challenge when analyzing cellular components is the preparation and isolation of these components. Detection of RNA was achieved by inductively coupled plasma mass spectrometry (ICP-MS), by quantifying the amount of ${ }^{31} \mathrm{P}^{+}$in the investigated sample [27]. However, the reported limit of detection was $\sim 60 \mathrm{pg}$, which is roughly 5 times larger than the expected quantity found within one cell. ICP-MS was also reported for quantification of proteins in cells, by quantifying the number of sulfur atoms [28]. However, the limit of detection was higher than $300 \mathrm{pg}$ of proteins [29], which is approximately 10 times higher than the values obtained for RMS. A major disadvantage of single-cell PCR and MS techniques is that they require cell lysis; thus, the cells are destroyed and cannot be used for subsequent analysis or imaging by other technique.

Although the AFM allows accurate measurements of cell thickness, such information is often impractical to obtain for live cells because of the long acquisition times, during which the cell morphology can change. Thus, in the absence of AFM topography data, the absolute values of molecular concentrations cannot be obtained. However, Figure 2 suggests that the image 
corresponding to the PC1 scores obtained from the PCA analysis of Raman maps resembles the height profile of the cell as measured by AFM (Figure 2(b)). This similarity is not unexpected because the PC1 loading represents the mean Raman spectrum of the cell. Thus, at a given position in the cell, the PC1 score is expected to be proportional to the cell mass and height. However, this assumption is valid when the cell thickness is smaller than the axial resolution of the laser spot, and thus the laser excitation intensity can be considered constant across the sampled cell volume.

To investigate the relationship between PC1 scores and cell height (measured by AFM) in more depth, we analyzed the PC1 images from Raman maps of five fixed cells.

(a)

AFM
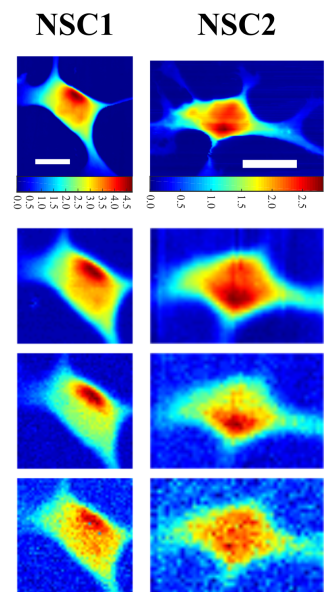
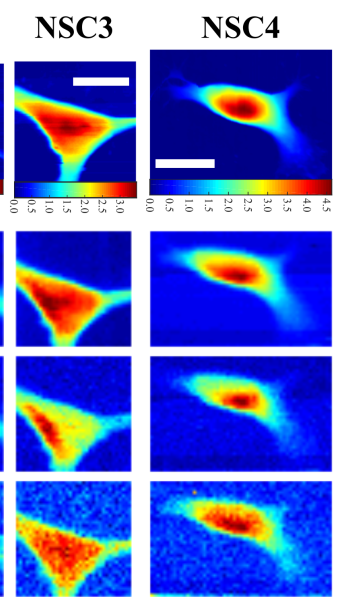
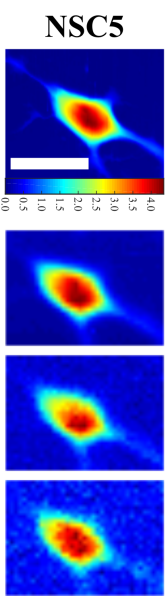

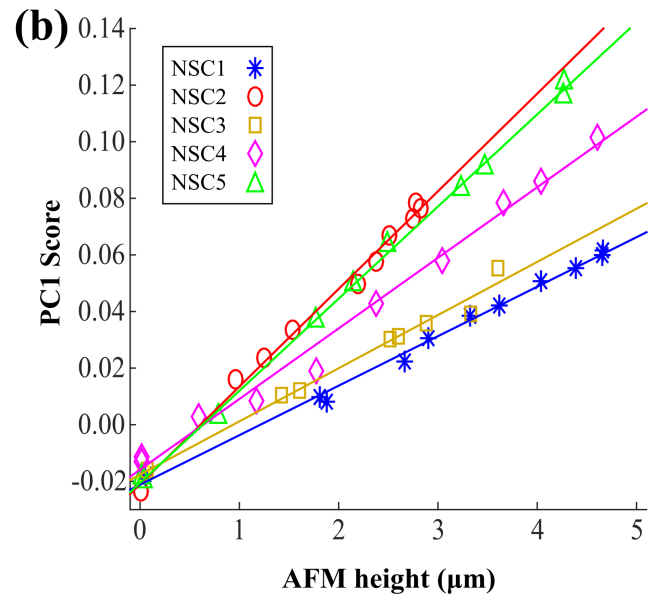

Figure 5. Cell normalization comparison. (a) shows AFM maps (scale bar $20 \mu \mathrm{m}$ ) for five different NSCs, with corresponding maps from Raman data, based on PC1 scores, and normalization by peak area for $1450 \mathrm{~cm}^{-1}$ and $1001 \mathrm{~cm}^{-1}$ peaks. (b) Correlation of AFM height with co-localized PC1 scores.

Figure 5(a) compares the AFM height profiles of five fixed cells and the maps corresponding to PC1 scores. The calculated correlation coefficients for the AFM and PC1 maps for the individual cells was above 0.99 , indicating that the PC1 scores from a Raman raster-scan can provide an accurate relative height profile for the cells. Figure 5(a) also shows that the map of PC1 scores is a more accurate representation of the cell topography that the previously used intensity of the $1450 \mathrm{~cm}-1$ band [12]. The use of the $1450 \mathrm{~cm}-1$ map can be convenient to use for normalization because it corresponds mainly to $\mathrm{CH}_{2}$ vibrations found in most biomolecules, but also it is an intense band for which the intensity can be calculated accurately. Nevertheless, Figure 5 indicates that the images corresponding to the $1450 \mathrm{~cm}^{-1}$ over-represent the lipid rich regions within the cells while under-representing the protein rich regions. Normalization to the $1001 \mathrm{~cm}^{-1}$ band (Figure 5) shows a better correlation to the AFM height map than the $1450 \mathrm{~cm}^{-1}$ normalization, however there is also significant noise from the background (errors in calculation of local baseline), which is avoided when using PC1. A regression analysis of the PC1 scores and AFM height at 9-11 randomly selected points within each of the five cells (Figure 5(b)), shows that, at each sampling point, the height of the cell is proportional to the PC1 score. However, 
Figure 5(b) shows that the slopes of the regression lines vary from cell to cell, therefore the maps of PC1 scores can provide only a relative profile of the cell topography.

We also investigated whether it is possible to obtain an absolute height profile of a cell by measuring the height of the cell at a single point using a Raman z-scan, and use this height value to scale the height profile obtained from PC1. In order to achieve this, we first calculated the axial point spread function $\left(\mathrm{PSF}_{\mathrm{z}}\right)$ of the Raman instrument by a de-convolution of the Raman zscans on fixed cells and the cell thickness values measured by AFM (modeled as a top-hat function). Figure 6(a) presents the 2D Raman maps corresponding to PC1, PC2 and PC3 at various values of $\mathrm{z}$. These results show that when the $\mathrm{z}$ position along the axial direction is changed, the Raman spectra images obtained by PCA analysis change in intensity but the general profiles remain the same. This indicates that the $\mathrm{PSF}_{\mathrm{z}}$ is significantly larger than the thickness of the cell.
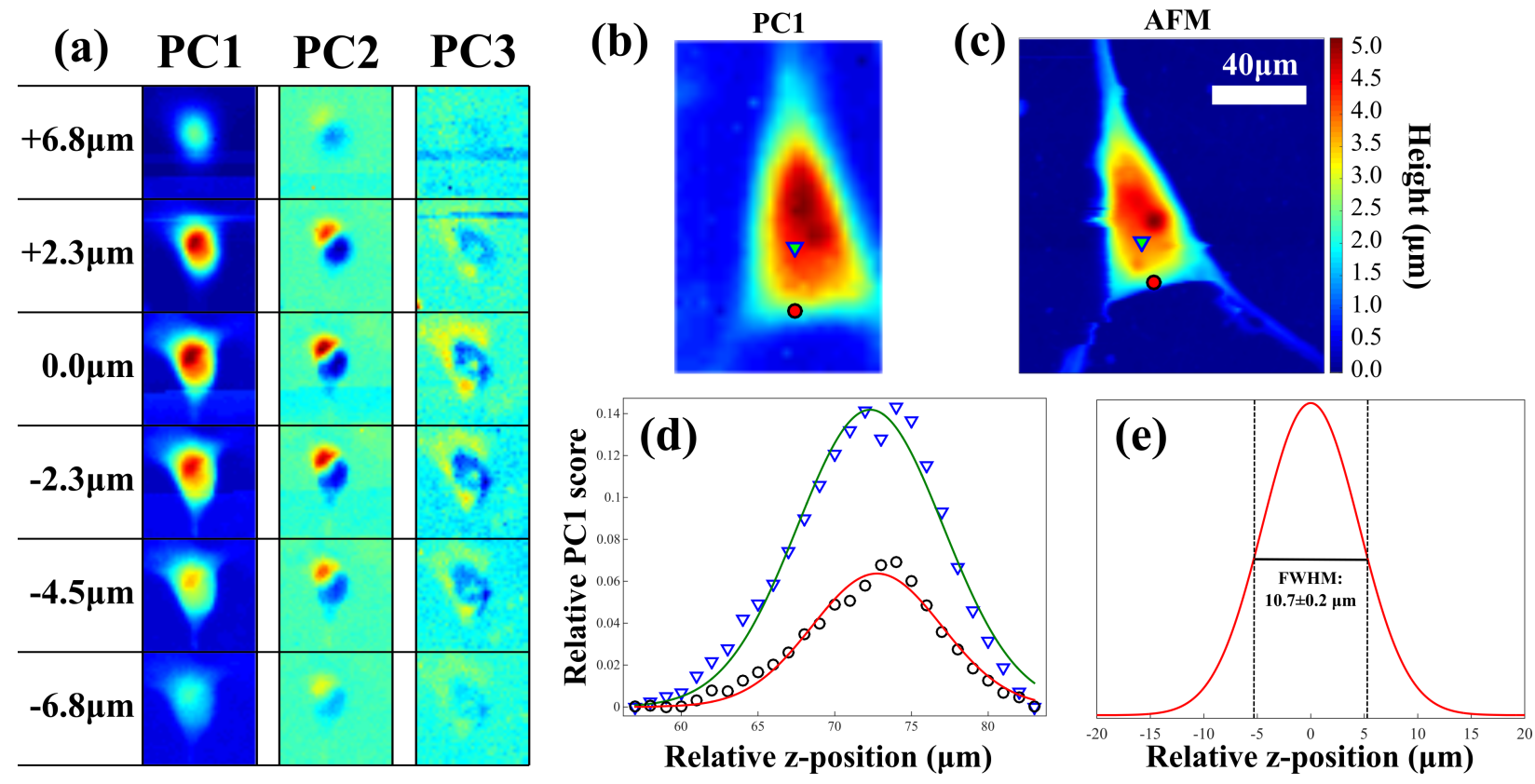

Figure 6. (a) Raman z-profiling images. (b) PC1 map and (c) AFM for an NSC for which 25 line scans at different z-positions were obtained. (d) shows the variation in the value of the PC1 score as the sampling z-position is varied at two locations on the cell of different thickness (indicated in (c) and (d)). Several PC1 z-profiles were fitted to a Gaussian function, matched to a top-hat function of width given by the corresponding AFM height, and de-convolved to obtain an estimate for the $\mathrm{PSF}_{\mathrm{z}}$. (e) shows the resulting average $\mathrm{PSF}_{\mathrm{z}}$ measured from 13 de-convolution measurements.

Figure 6(d) presents a typical z-profiles of the PC1 scores, obtained at two positions in a fixed cell. These z-profiles represent the convolution of the $\mathrm{PSF}_{\mathrm{z}}$ of the instrument and the thickness of the cell at the corresponding positions. As the height of the cells at these positions were 
measured by AFM, the $\mathrm{PSF}_{\mathrm{z}}$ of the instrument was calculated by de-convolution, obtaining a Gaussian function with FWHM of $10.7 \pm 0.2 \mu \mathrm{m}$ (data from 3 cells were used, with 13 Raman zscans for each cell). This measured FWHM was larger than the theoretical value of $1.45 \mu \mathrm{m}$, discrepancies likely to be due to the use of $\mathrm{MgF}_{2}$ rather than a glass/quarz coverslip, as well as potential differences in refractive index variations within the cells. After the calculation of the $\mathrm{PSF}_{\mathrm{z}}$, Raman z-scans obtained from five new cells were de-convolved in order to obtain the local height of the cells. However, comparison between the height values obtained by deconvolution and the values measured by AFM indicated typical errors of $\sim 50 \%$. The main factor affecting these errors is related to the fact that the FWHM of the $\mathrm{PSF}_{\mathrm{z}}$ is much larger than the typical heights of the cells $(\sim 1-5 \mu \mathrm{m})$. Using a confocal Raman microscope with a narrower PSF $_{z}$ may improve the measurement of the cell height. However, measuring 2D Raman maps with a narrower $\mathrm{PSF}_{\mathrm{z}}$ would create $\mathrm{PC} 1$ map that are no longer representative to cell thickness as the laser intensity in the axial direction is no longer constant within throughout the cell thickness. Thus, a full 3D Raman scan would be required in order to measure the cell topography and obtain absolute molecular concentration maps of the cell.

Because the acquisition of 3D Raman maps are impractical, in particular for live cells, we measured 2D Raman maps of live NSCs and obtained relative concentration maps for RNA and proteins by applying the calibration models in Figure 3 and normalizing the Raman spectra using the PC1 scores. Figure 7 shows that, after normalization, the concentration of proteins in the cells is homogenous, which is in agreement with the results for fixed cells. This result is to be expected, especially considering that the acquisition time of the Raman maps was 5-15 minutes, averaging any potential short-lived heterogeneities. On the other hand, RNA distribution indicates a higher concentration of RNA in the cytoplasm than in the nucleus and the endoplasmic reticulum, in agreement with the results for fixed cells, and expected biologically.

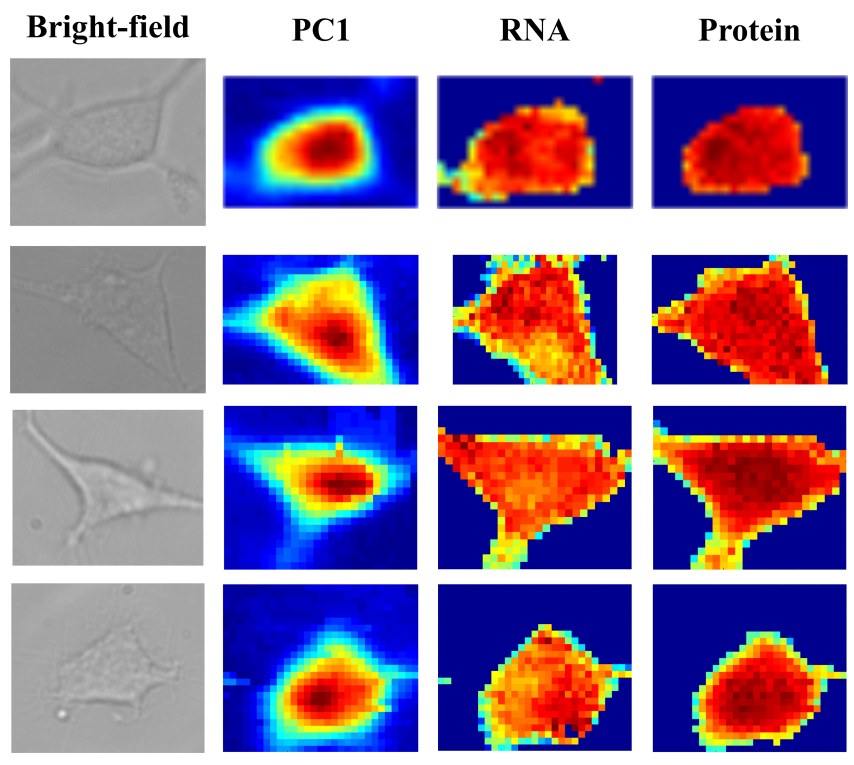

Figure 7: Relative concentration of RNA and proteins in live NSCs. The concentration maps were obtained by applying the calibration models described in Figure 3 on Raman maps normalized using the scores of PC1. 


\section{Conclusion}

RMS and AFM are non-invasive label-free techniques that can be used on cells to provide complementary information. RMS mapping allows qualitative measurements of the spatial distribution of particular biomolecules in living cells. However, when comparing cells with different morphologies, it is difficult to know whether spectral differences arise from molecular differences (i.e. differences in concentration) or are due to cell topography. Here, we combined RMS with AFM to understand these effects. AFM was used to map the topography of fixed cells, and thus provide a representation of cell volume. Using solution-based calibration models for RNA and albumin, the RMS and AFM data was used to create quantitative maps of RNA and proteins in individual fixed cells. Because the acquisition times of AFM and Raman measurements are too long to be compatible with live cells measurements, we also used the combined AFM-Raman dataset from fixed cells to investigate potential improvements for normalization of Raman spectral maps. We found that if the PSF of the Raman instrument is significantly wider than the thickness of the cells, the maps of Raman PC1 scores can be correlated with the AFM map, and thus the cell volume. Using this technique, qualitative maps of RNA and protein concentrations were obtained for live cells. While this study demonstrates the potential of using AFM and RMS for measuring concentration maps for individual NSCs invitro, further studies are required to establish the robustness of the normalization method based on principal component analysis when comparing Raman spectra of cells with large morphological differences.

\section{Acknowledgements}

The development of the Raman micro-spectrometer was supported by the Biotechnology and Biological Sciences Research Council UK research grant BB/G010285/1. The NSC culture was available thanks to support from the Alzheimer's Society (S.S. and V.S.).

\section{References}

[1] Larissa Mueller, Heike Traub, Norbert Jakubowski, Daniela Drescher, Vladimir I. Baranov, and Janina Kneipp. "Trends in single-cell analysis by use of ICP-MS." Analytical and bioanalytical chemistry 406, no. 27 (2014): 6963-6977.

[2]Anders Ståhlberg, and Mikael Kubista. "The workflow of single-cell expression profiling using quantitative real-time PCR." Expert review of molecular diagnostics 14, no. 3 (2014): 323331. 
[3] G. J. Puppels, F. F. M. De Mul, C. Otto, J. Greve, M. Robert-Nicoud, D. J. Arndt-Jovin, and T. M. Jovin. "Studying single living cells and chromosomes by confocal Raman microspectroscopy." Nature (1990): 301-303.

[4] Almar F. Palonpon, Mikiko Sodeoka, and Katsumasa Fujita. "Molecular imaging of live cells by Raman microscopy." Current opinion in chemical biology 17, no. 4 (2013): 708-715.

[5] Thomas Huser, and James Chan. "Raman spectroscopy for physiological investigations of tissues and cells." Advanced drug delivery reviews 89 (2015): 57-70.

[6] Birthe Kann, Herman L. Offerhaus, Maike Windbergs, and Cees Otto. "Raman microscopy for cellular investigations-From single cell imaging to drug carrier uptake visualization." Advanced drug delivery reviews (2015).

[7] Masaya Okada, Nicholas Isaac Smith, Almar Flotildes Palonpon, Hiromi Endo, Satoshi Kawata, Mikiko Sodeoka, and Katsumasa Fujita. "Label-free Raman observation of cytochrome c dynamics during apoptosis." Proceedings of the National Academy of Sciences 109, no. 1 (2012): 28-32.

[8] Hemanth Nag Noothalapati Venkata, and Shinsuke Shigeto. "Stable isotope-labeled Raman imaging reveals dynamic proteome localization to lipid droplets in single fission yeast cells." Chemistry \& biology 19, no. 11 (2012): 1373-1380.

[9] Flavius C. Pascut, Spandan Kalra, Vinoj George, Nathan Welch, Chris Denning, and Ioan Notingher. "Non-invasive label-free monitoring the cardiac differentiation of human embryonic stem cells in-vitro by Raman spectroscopy." Biochimica et Biophysica Acta (BBA)-General Subjects 1830, no. 6 (2013): 3517-3524.

[10] Abida Naemat, Hany M. Elsheikha, Alaa Al-Sandaqchi, Kenny Kong, Adrian Ghita, and Ioan Notingher. "Analysis of interaction between the apicomplexan protozoan Toxoplasma gondii and host cells using label-free Raman spectroscopy." Analyst 140, no. 3 (2015): 756-764.

[11] Stanislav O. Konorov, H. Georg Schulze, Chad G. Atkins, James M. Piret, Samuel A. Aparicio, Robin FB Turner, and Michael W. Blades. "Absolute quantification of intracellular glycogen content in human embryonic stem cells with Raman microspectroscopy." Analytical chemistry 83, no. 16 (2011): 6254-6258.

[12] Adrian Ghita, Flavius C. Pascut, Melissa Mather, Virginie Sottile, and Ioan Notingher. "Cytoplasmic RNA in undifferentiated neural stem cells: a potential label-free Raman spectral marker for assessing the undifferentiated status." Analytical chemistry 84, no. 7 (2012): 31553162 .

[13] I. Notingher, S. Verrier, S. Haque, J. M. Polak, and L. L. Hench. "Spectroscopic study of human lung epithelial cells (A549) in culture: living cells versus dead cells." Biopolymers 72, no. 4 (2003): 230-240. 
[14] Yong Chen Su, Le, G. N. Zhang, L. H. Wang, A. G. Shen, X. D. Zhou, X. H. Wang, and J. $\mathrm{M}$. Hu. "In vivo and in situ monitoring of the nitric oxide stimulus response of single cancer cells by Raman spectroscopy." Laser Physics Letters 10, no. 4 (2013): 045608.

[15] Quinn Matthews, Andrew Jirasek, Julian Lum, Xiaobo Duan, and Alexandre G. Brolo. "Variability in Raman spectra of single human tumor cells cultured in vitro: correlation with cell cycle and culture confluency." Applied spectroscopy 64, no. 8 (2010): 871-887.

[16] Mingzhou Chen, Naomi McReynolds, Elaine C. Campbell, Michael Mazilu, João Barbosa, Kishan Dholakia, and Simon J. Powis. "The Use of Wavelength Modulated Raman Spectroscopy in Label-Free Identification of T Lymphocyte Subsets, Natural Killer Cells and Dendritic Cells." PLoS ONE (2015): e 0125158.

[17] Flavius C. Pascut, Huey T. Goh, Nathan Welch, Lee D. Buttery, Chris Denning, and Ioan Notingher. "Noninvasive detection and imaging of molecular markers in live cardiomyocytes derived from human embryonic stem cells." Biophysical journal 100, no. 1 (2011): 251-259.

[18] Tatiana Tolstik, Claudio Marquardt, Christian Matthäus, Norbert Bergner, Christiane Bielecki, Christoph Krafft, Andreas Stallmach, and Jürgen Popp. "Discrimination and classification of liver cancer cells and proliferation states by raman spectroscopic imaging." Analyst 139, no. 22 (2014): 6037-6044.

[19] Samir F. El-Mashtoly, Hesham K. Yosef, Dennis Petersen, Laven Mavarani, Abdelouahid Maghnouj, Stephan Hahn, Carsten Kötting, and Klaus Gerwert. "Label-Free Raman Spectroscopic Imaging Monitors the Integral Physiologically Relevant Drug Responses in Cancer Cells." Analytical chemistry 87, no. 14 (2015): 7297-7304.

[20] Stephen C. Erfurth, Ernest J. Kiser, and Warner L. Peticolas. "Determination of the backbone structure of nucleic acids and nucleic acid oligomers by laser Raman scattering." Proceedings of the National Academy of Sciences 69, no. 4 (1972): 938-941.

[21] J. M. Benevides, and G. J. Thomas. "Characterization of DNA structures by Raman spectroscopy: high-salt and low-salt forms of double helical poly (dG-dC) in $\mathrm{H} 2 \mathrm{O}$ and $\mathrm{D} 2 \mathrm{O}$ solutions and application to B, Z and A-DNA*." Nucleic acids research 11, no. 16 (1983): 57475761.

[22] Thomas G. Spiro, and Bruce P. Gaber. "Laser Raman scattering as a probe of protein structure." Annual review of biochemistry 46, no. 1 (1977): 553-570.

[23] Christoph Krafft, Lars Neudert, Thomas Simat, and Reiner Salzer. "Near infrared Raman spectra of human brain lipids." Spectrochimica Acta Part A: Molecular and Biomolecular Spectroscopy 61, no. 7 (2005): 1529-1535.

[24] Ron Milo. "What is the total number of protein molecules per cell volume? A call to rethink some published values." Bioessays 35, no. 12 (2013): 1050-1055. 
[25] C. Chen, D. A. Ridzon, A. J. Broomer, Z. Zhou, D. H. Lee, J. T. Nguyen, M. Barbisin, N. L. $\mathrm{Xu}$, V. R. Mahuvakar, M. R. Andersen, K. Q. Lao, K. J. Livak, K. J. Guegler. "Real-time quantification of microRNAs by stem-loop RT-PCR." Nucleic Acids Research 33, no 20 (2005): e179.

[26] G.A. Valaskovic, N. L. Kelleher, F. W. McLafferty FW. "Attomole protein characterization by capillary electrophoresis-mass spectrometry”. Science 273 (1996):1199-1202)

[27] Shin-ichiro Fujii, Kazumi Inagaki, Shin-ichi Miyashita, Alexander S. Groombridge, Keisuke Nagasawa, Koichi Chiba and Akiko Takatsu. "Separation and quantification of RNA molecules using size-exclusion chromatography hyphenated with inductively coupled plasmamass spectrometry." Electrophoresis 35, no 9 (2014): 1315-1318.

[28] M. Wind, A. Wegener, A. Eisenmenger, R. Kellner, W. D. Lehmann. "Sulfur as the Key Element for Quantitative Protein Analysis by Capillary Liquid Chromatography Coupled to Element Mass Spectrometry." Angewandte Chemie International Edition 42, no 29 (2003): 34253427.

[29] Nico Zinn, Ralf Krüger, Peter Leonhard, Jörg Bettmer. " $\mu$ LC coupled to ICP-SFMS with post-column isotope dilution analysis of sulfur for absolute protein quantification." Analytical and Bioanalytical Chemistry 391(2): 537-543. 